Questions vives

\section{Questions Vives}

Recherches en éducation

$n^{\circ} 22 \mid 2014$

Questionner le curriculum en éducation physique et sportive : Quelle dynamique en contexte? Quelle autonomie?

\title{
Le curriculum en actes révélé par les gestes didactiques de métier : étude des pratiques didactiques d'un enseignant débutant en EPS
}

The curriculum in acts revealed by the "gestes didactiques de métier": study of the didactic practices of a novice teacher in PE

Fabienne Brière-Guenoun

\section{(2) OpenEdition}

\section{Journals}

Édition électronique

URL : http://journals.openedition.org/questionsvives/1623

DOI : $10.4000 /$ questionsvives. 1623

ISSN : $1775-433 \mathrm{X}$

Éditeur

Université Aix-Marseille (AMU)

Édition imprimée

Date de publication : 15 décembre 2014

ISBN : 978-2-912643-46-9

ISSN : 1635-4079

Référence électronique

Fabienne Brière-Guenoun, « Le curriculum en actes révélé par les gestes didactiques de métier : étude des pratiques didactiques d'un enseignant débutant en EPS », Questions Vives [En ligne], n² 22 | 2014, mis en ligne le 16 février 2015, consulté le 19 avril 2019. URL : http://journals.openedition.org/ questionsvives/1623 ; DOI : 10.4000/questionsvives.1623

Ce document a été généré automatiquement le 19 avril 2019.

\section{(i) $\$$}

Questions Vives est mis à disposition selon les termes de la licence Creative Commons Attribution -

Pas d'Utilisation Commerciale - Pas de Modification 4.0 International. 


\title{
Le curriculum en actes révélé par les gestes didactiques de métier : étude des pratiques didactiques d'un enseignant débutant en EPS
}

\author{
The curriculum in acts revealed by the "gestes didactiques de métier": study of \\ the didactic practices of a novice teacher in PE
}

Fabienne Brière-Guenoun

\section{Introduction}

1 Les enjeux d'une refondation du système scolaire en France ${ }^{1}$ interrogent non seulement les contenus disciplinaires mais aussi les conditions de leur mise en place par le professeur dans sa classe. Selon une approche élargie des problématiques curriculaires, les transformations en matière d'apprentissage des élèves relèvent du curriculum effectif, qui renvoie à la concrétisation en contexte du curriculum prescrit, officiel ou formel (Audigier \& Tuteaux-Guillon, 2008). Par extension, le curriculum en actes rend compte des actions effectivement déployées par le professeur et les élèves pour mettre en œuvre le curriculum effectif. Il désigne donc les manières dont sont co-construits les savoirs dans la classe en connexion avec les prescriptions officielles, en particulier les programmes de la discipline, mais également avec les pratiques sociales dont ils sont issus (Schubauer-Leoni \& Leutenegger, 2005).

2 Afin de rendre compte de la dynamique du curriculum en actes, nous nous intéressons aux manières dont le professeur, conjointement à l'activité des élèves, élabore, négocie et remanie les contenus d'enseignement dans la classe en lien avec les références externes et les prescriptions officielles. Autrement dit, les "gestes didactiques du professeur ", rattachés aux actions qu'il déploie in situ pour conduire l'étude des élèves, constituent un 
indicateur du curriculum en actes. Centrée plus particulièrement sur les pratiques didactiques d'un enseignant stagiaire en éducation physique et sportive (EPS), notre recherche vise également à documenter les façons singulières dont le professeur débutant s'approprie le métier lors de son insertion professionnelle. Nous inscrivant dans une approche comparatiste en didactique (Schubauer-Leoni, 2008; Sensevy \& Mercier, 2007), nous souhaitons ainsi saisir les traits spécifiques et génériques des "gestes didactiques de métier » du professeur débutant en tant qu'indicateurs des manières dont il met en actes le curriculum.

\section{Cadre théorique}

Dans cette section, nous nous attachons à définir le concept de « gestes didactiques de métier » afin d'expliciter ce en quoi il permet de renseigner le curriculum en actes et ce qui détermine ce dernier.

\subsection{Des actes ancrés dans l'agir conjoint}

4 Selon la théorie de l'action conjointe en didactique (Sensevy \& Mercier, 2007), le système des gestes du professeur et celui des élèves se déterminent mutuellement lors de tâches coopératives en vue d'établir une référence commune à propos des enjeux de savoir. Au sein de cette communauté d'étude, «le rôle du professeur consiste non seulement à organiser un chemin pour l'apprentissage de ses élèves mais aussi à les aider à faire ce "bout de chemin" qui correspond au temps de l'étude » (Brière-Guenoun \& Refuggi, 2007, p. 68). Ainsi, les gestes du professeur relèvent de deux types de tâches professorales visant à conduire l'avancée des savoirs dans la classe (chronogenèse): des tâches d'organisation et de conception des dispositifs d'apprentissage; des tâches d'aide à l'étude (Chevallard, 1997). Ces tâches se rattachent à l'élaboration d'une référence commune dont l'enjeu est de faire vivre les savoirs dans la classe en connexion avec les pratiques sociales dont ils proviennent (Schubauer-Leoni, Leutenegger, Ligozat \& Flückiger, 2007). Elles renvoient donc au curriculum en actes qui caractérise les façons dont les savoirs étudiés prennent en considération et concilient les références externes, les prescriptions officielles, les intentions et préoccupations didactiques du professeur ainsi que l'activité d'apprentissage des élèves.

5 Les procédés par lesquels se co-construisent les savoirs, nommés « analyseurs de l'action conjointe » (Amade-Escot, 2013) peuvent être étudiés selon trois plans :

- un plan mésogénétique qui renvoie aux processus de construction du milieu didactique, caractérisant l'ensemble des objets matériels, symboliques, conceptuels introduits à des fins d'élaboration d'un système commun de significations entre le professeur et les élèves ;

- un plan chronogénétique qui caractérise la gestion des temps d'apprentissage et d'enseignement ;

- un plan topogénétique qui concerne les manières dont sont partagées les responsabilités entre élèves et professeur dans les transactions didactiques, renvoyant à la gestion des phases de l'activité des élèves et des positions (places) occupées par les différents acteurs.

Selon ce modèle, les gestes de l'enseignant se déclinent en quatre types d'actions inscrites dans la relation didactique: a) définir les enjeux d'apprentissage; b) réguler ou gérer l'incertitude en guidant l'activité d'apprentissage des élèves; c) dévoluer ou rendre les 
élèves responsables de leur appropriation des savoirs; et d) institutionnaliser (ou instituer), c'est-à-dire légitimer certaines réponses des élèves.

7 Ainsi, parce que les gestes du professeur désignent des actions spécifiques liées aux tâches professorales, interdépendantes des gestes d'étude des élèves et des enjeux de savoir, ils donnent accès aux modalités dont les savoirs sont mis à l'étude et étudiés.

\subsection{La réflexivité dans et sur l'action, un moyen d'accès au curriculum en actes}

$8 \quad$ Les gestes du professeur traduisent d'autre part ses modalités d'analyse et de réflexivité dans et sur l'action qui documentent le raisonnement pratique initiant l'action in situ et les manières dont ce dernier s'enrichit au fil de l'expérience par une réflexivité a posteriori sur l'action.

9 Ce faisant, la mise au jour des conflits de préoccupations à travers ce raisonnement articulé aux pratiques renseigne les activités suspendues, contrariées, empêchées (Amigues, 2003, 2009 ; Félix \& Saujat, 2008 ; Monnier \& Amade-Escot, 2009) qui définissent le «réel de l'activité" au sens de Clot (1999). La fonction attribuée à ces modalités d'analyse et de réflexivité dans la compréhension du curriculum en actes est bien d'éclairer les focalisations du professeur, les conflits et compromis éventuels portant sur les dimensions épistémiques de son activité. En EPS, les recherches montrent que les démarches d'analyse des situations vécues en classe sont centrées sur l'interprétation de l'activité d'apprentissage des élèves (Grosstephan, 2010; Leriche, 2010; Vinson \& Dugal, 2011) et reposent sur différents registres - narratif, descriptif, explicatif, interprétatif (Amade-Escot \& Dugal, 2010 ; Brière-Guenoun \& Musard, 2012). Grosstephan (2010) met en évidence deux "modalités d'analyse et de réflexivité » de professeurs d'EPS en formation continue : l'une mobilisant un raisonnement logique visant à justifier ses choix théoriques, l'autre centrée sur l'interprétation des conduites effectives des élèves en vue d'expliquer et valider empiriquement ses choix.

10 L'étude des modalités d'analyse et de réflexivité permet donc d'éclairer les préoccupations didactiques du professeur et les tensions qui l'animent, révélant par làmême les choix qu'il effectue pour mettre en œuvre le curriculum.

\subsection{Les gestes déterminés par l'épistémologie pratique du professeur}

11 Les gestes du professeur résultent ainsi de préoccupations multiples, imbriquées et parfois contradictoires concernant le choix des objets de savoirs, les façons dont ils sont mis en scène dans les tâches et les modalités de conduite des apprentissages. Ces préoccupations traduisent les motifs d'agir singuliers du professeur et ses intentions didactiques (préalables), relevant de son projet d'enseignement.

12 Au-delà de préoccupations circonscrites dans des situations temporellement délimitées, les gestes du professeur actualisent son "épistémologie pratique ", désignant "une théorie plus ou moins implicite de la connaissance (des savoirs enseignés), de sons sens, de son usage, des relations que telle connaissance entretient avec telle autre » (Sensevy, 2007, p. 37). Cette théorie ancrée dans la pratique oriente l'« étayage didactique »du professeur (Toullec-Théry \& Marlot, 2013, p. 51) et reflète les manières dont ce dernier 
prélève des indices sur l'activité des élèves et les exploite (ou non) pour faire jouer le jeu didactique (Amade-Escot, 2013). Au regard de notre projet de comprendre et d'expliquer les manières dont se co-construit en actes le curriculum, ce concept renseigne les dimensions contextualisée, dynamique et interactive des savoirs enseignés et les filiations qu'entretiennent ces derniers avec les références externes. L'épistémologie pratique du professeur résulte de ses expériences au sein des communautés ou institutions où il a rencontré les savoirs enseignés et agit comme un déterminant du jeu didactique (Sensevy, 2007). En EPS, les travaux montrent qu'elle s'enracine dans les «assujettissements » de l'enseignant (Chevallard, 2003) vis-à-vis des diverses institutions de formation et d'exercice professionnel qui orientent ses façons de mettre en scène les enjeux de savoirs (Amade-Escot, Amans-Passaga \& Montaud, 2009; Brière-Guenoun \& Amade-Escot, 2010 ; Devos-Prieur, 2011).

En conséquence, l'étude des " gestes didactiques de métier » rend compte des façons dont vivent les savoirs dans la classe en lien avec le(s) rapport(s) aux savoirs et le rapport au métier du professeur. De façon analogique avec le concept de rapport au savoir, nous désignons par rapport au métier l'ensemble des relations de sens qu'établit le professeur avec son métier dans ses dimensions personnelles, impersonnelles, interpersonnelles et transpersonnelles (au sens de Clot, 2008, 2013). Le rapport au métier dépend des diverses expériences et appartenances institutionnelles de l'enseignant et relève des manières dont il s'empare du genre du métier, le (re)définit, le façonne en construisant son propre style qui en retour participe de l'évolution du genre.

En résumé, les "gestes didactiques de métier " traduisent l'épistémologie pratique du professeur et son rapport au métier, à l'origine de la construction du jeu didactique avec les élèves.

\subsection{Problématique: des gestes didactiques de métier au curriculum en actes}

15 Finalement, notre recherche vise à caractériser le curriculum en actes à partir des " gestes didactiques de métier » du professeur. Par « gestes didactiques de métier » nous désignons les actions spécifiques du professeur lui permettant de conduire l'étude des élèves en une institution donnée (Chevallard, 1999). Imbriqués aux gestes d'étude des élèves, ils actualisent en contexte les intentions et préoccupations didactiques du professeur et révèlent son épistémologie pratique, elle-même pour partie dévoilée par ses modalités d'analyse et de réflexivité dans et sur l'action. Ils sont déterminés par les prescriptions, les normes véhiculées par l'école ou la société et par les pré-construits historico-culturels que constituent les savoirs et leur cristallisation dans les œuvres désignées pour être mises à l'étude (Schubauer-Leoni et al., 2007).

16 Notre projet consiste à caractériser puis comparer au cours de deux leçons menées à différents moments de l'année les gestes didactiques de métier d'un enseignant stagiaire (ES) afin de comprendre les processus à l'œuvre dans l'élaboration du curriculum en actes et ce qui les détermine. L'étude des gestes didactiques de métier procède de trois étapes : a) l'identification des objets de savoirs mis à l'étude et le rôle des élèves au fil du temps, liés aux tâches d'organisation et de conception des dispositifs (chronogenèse et topogenèse) ; b) l'analyse des processus de construction du milieu didactique, liés aux tâche d'aide à l'étude (mesogenèse); et c) la caractérisation de l'épistémologie pratique 
du professeur en lien avec les déterminants de l'action didactique d'ordre institutionnel et culturel.

\section{Méthode}

Pour rendre compte du curriculum en actes tel qu'il s'actualise dans les gestes didactiques de métier du professeur débutant, nous exploitons les descripteurs de l'action conjointe selon une analyse ascendante de la transposition didactique (Schubauer-Leoni \& Leutenegger, 2005) croisant les points de vue intrinsèque et extrinsèque. La démarche méthodologique, inspirée de l'«approche clinique et expérimentale du didactique ordinaire" (Leutenegger, 2009), respecte les principes de rétroaction des analyses, de questionnements réciproques et de symétrie entre les différentes traces.

\subsection{Contexte, participants à l'étude et dispositif méthodologique}

L'ES, que nous appelons Grégoire, enseigne en lycée et a choisi d'être observé avec une classe de première scientifique, constituée de 25 élèves. Il a participé à l'étude durant une année scolaire (correspondant à l'année de stage) à partir de mi-octobre ${ }^{2}$ jusqu'à la fin de l'année.

Le dispositif méthodologique, synthétisé dans la figure 1, repose sur : a) l'enregistrement filmé de la deuxième leçon et la cinquième d'un cycle d'escalade et de la troisième leçon d'un cycle de volley-ball ${ }^{3}$; b) des entretiens de type auto-confrontation, menés après chaque leçon filmée ; c) deux entretiens semi-directifs, réalisés respectivement en début (entretien ante-cycle) et en fin d'année scolaire (entretien post-recueil).

Figure 1 : Synthèse du dispositif méthodologique.

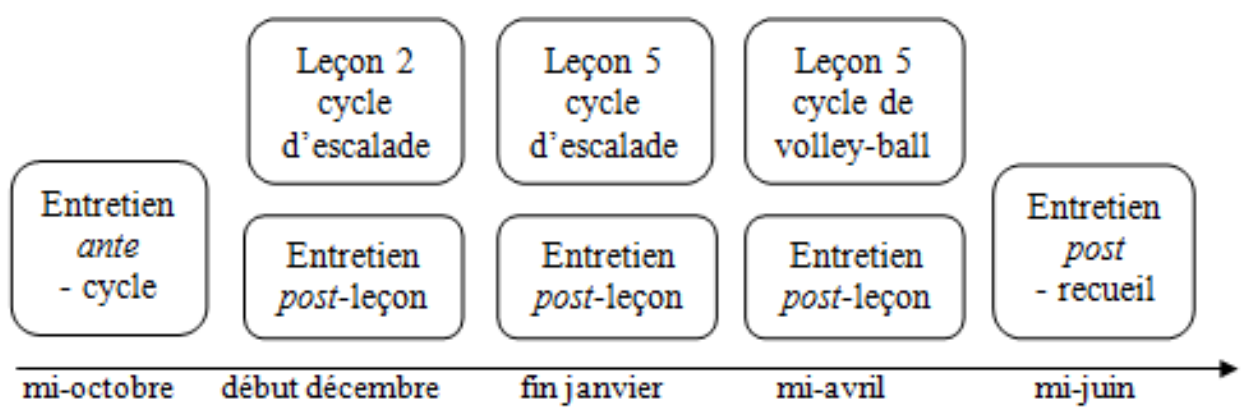

Les enregistrements filmés suivent les déplacements de l'ES au cours des moments clés de la leçon. Leur fonction est de documenter le point de vue extrinsèque (du chercheur) en mettant en évidence le découpage temporel de la leçon. Les entretiens post-leçons, de type auto-confrontation, prennent appui sur différentes séquences filmées (retenues par le chercheur) typiques des différents moments clés de la leçon, à savoir l'échauffement, la présentation des tâches, les interventions individuelles et collectives de l'ES auprès des élèves et le bilan de séance. Inspirés des méthodes développées en ergonomie (Clot, 1999) mais spécifiés au regard des enjeux didactiques de la recherche, leur fonction dans le dispositif méthodologique est de documenter le point de vue intrinsèque (de l'enseignant), de façon similaire aux entretiens post tels que définis par Leutenegger (2009). Ces entretiens visent à mettre au jour les préoccupations de l'enseignant du point 
de vue des dispositifs (mis à l'étude, envisagés, voire évacués), des réaménagements du milieu didactique (opérés ou envisagés) en lien avec les façons dont il analyse les enjeux de savoir et les conduites d'apprentissage des élèves. Ils sont envisagés comme techniques d'accès contrôlé par le chercheur aux "raisons » des gestes de l'ES et au-delà aux manières dont il entre en résonance avec divers aspects du métier (caractérisant son rapport au métier).

21 L'entretien ante-cycle concerne les différents éléments du contexte tels qu'analysés par Grégoire et son projet d'enseignement pour la classe. L'entretien post-recueil se focalise sur le rapport au métier de l'ES en l'amenant à prendre du recul vis-à-vis de ses interventions lors de leçons spécifiques. Les entretiens ante-cycle et post-recueil, afférents au point de vue intrinsèque, visent à compléter, confirmer ou infirmer les interprétations faites à partir du corpus principal (enregistrements filmés et entretiens de type autoconfrontation).

\subsection{Traitement des données et organisation des analyses}

La chronologie des analyses des différents corpus procède d'une démarche ascendante qui va de l'analyse interne à l'analyse externe de la leçon (Leutenegger, 2009).

Le premier temps de l'analyse consiste à construire le synopsis de chaque leçon (à partir des enregistrements audio-visuels) afin de retracer les différents types de tâches mis à l'étude en fonction des actions de l'enseignant (définir, reguler, dévoluer, institutionnaliser), des objets de savoir enjeux des dispositifs et des places respectives du professeur et des élèves (niveau mésoscopique).

Dans un second temps, nous extrayons $d u$ corpus des «évènements remarquables » (niveau microscopique), articulant des données filmées et d'entretien post -leçon ${ }^{4}$, à des fins d'analyse des processus en jeu dans la mise en actes du curriculum. "Considérés comme des moments cruciaux et emblématiques de la séance, relativement au questionnement de recherche » (Schubauer-Leoni \& Leutenegger, 2002, p. 246), les évènements remarquables sont choisis en fonction de leur capacité à rendre compte des modalités interactives de construction des savoirs au regard de la chronogenèse, de la mésogenèse et/ou de la topogenèse. Ils mettent en relation les gestes didactiques de l'ES, rapportés à l'activité des élèves (leurs conduites et verbalisations), avec ses intentions et préoccupations didactiques, révélées par ses modalités d'analyse et de réflexivité dans les entretiens post-leçons.

Dans un dernier temps, les indices prélevés dans les entretiens ante-cycle et post-recueil complètent les interprétations du chercheur et permettent de renseigner les façons dont le professeur s'approprie les prescriptions et les dimensions du métier (niveau macroscopique).

En résumé, le croisement des points de vue (intrinsèque et extrinsèque) selon plusieurs échelles temporelles vise à documenter le curriculum en actes à travers l'analyse du « réel » (Clot, 1999) des « gestes didactiques de métier » de l’ES.

\section{Résultats}

Pour chaque leçon observée, nous décrirons les gestes didactiques de Grégoire dans les tâches d'organisation et de conception des dispositifs puis dans les tâches d'aide à l'étude 
en lien avec leurs fonctions chronogénétiques, topogénétiques et mésogénétiques. Nous procéderons ensuite à l'analyse comparative de la mise en actes du curriculum au cours des deux leçons.

\subsection{Les gestes de Grégoire lors de la leçon d'escalade}

\subsubsection{Les gestes rattachés aux tâches d'organisation et de conception des dispositifs d'étude}

Le synopsis de la leçon d'escalade (tableau 1), la seconde du cycle, permet d'identifier des actes (Sensevy, 2007) correspondant aux différents types de tâches mis en place par Grégoire (tableau 1). Il met en évidence le découpage chronologique des objets de savoirs mis à l'étude (chronogenèse) et le mode de partage des responsabilités entre enseignant et élèves vis-à-vis des enjeux de savoir (topogenèse).

Tableau 1 : Synopsis de la leçon 2 d'escalade.

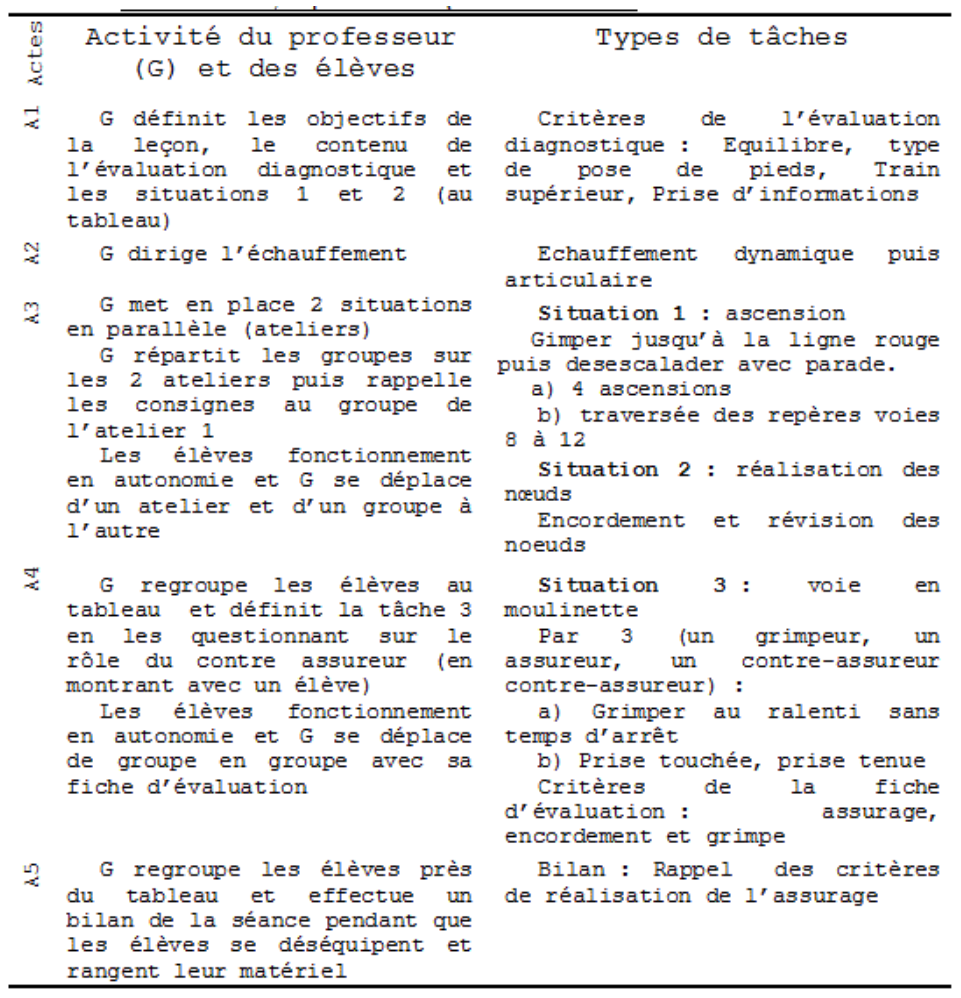

29 Le synopsis montre que la leçon se structure autour de trois types de tâches (deuxième colonne du tableau 1) : les deux premiers, proposés sous forme d'ateliers, sont consacrés respectivement aux techniques de grimpe et d'assurage; le troisième concerne la réalisation d'une voie en moulinette avec un assureur et un contre-assureur. L'analyse a priori des types de tâches mis à l'étude montre que les enjeux de savoir concernent l'assurage, les déplacements - appuis pieds et enchaînement, anticipation des appuis et prises - et la réalisation d'une voie en moulinette. Ils sont liés aux intentions didactiques de Grégoire qui visent à utiliser les différentes parties du pied, se décoller de la paroi, anticiper son déplacement pour aller vers le "grimper en tête" (entretien post-leçon).

Le mode organisationnel adopté par Grégoire (première colonne du tableau 1) consiste à définir la tâche puis à s'assurer, en circulant de groupe en groupe, que chaque élève 
s'engage bien dans le travail demandé (Actes 3 et 4). Dans la tâche 3, il définit particulièrement le rôle du contre assureur en réalisant une démonstration du geste avec un élève qu'il désigne (Acte 4). Sur le plan topogénétique, on constate ainsi une responsabilité différenciée du professeur et des élèves vis-à-vis des savoirs: alors que Grégoire prend à sa charge la définition des tâches, la désignation, l'institutionnalisation et l'évaluation des comportements attendus sur le plan sécuritaire (en renseignant son livret d'évaluation lors de la tâche 3), il leur délègue la responsabilité du choix de la voie et de l'organisation du travail au sein de la triplette (Acte 4).

\subsubsection{L'aide à l'étude pilotée par les objets de savoir concernant l'assurage}

31 L'agencement du milieu didactique relève de la mise en place de dispositifs juxtaposant (dans les ateliers) puis associant (au sein de la tâche de référence) les objets de savoirs (liés aux rôles de grimpeur et d'assureur). L'analyse des interactions didactiques révèle que c'est essentiellement le thème de l'assurage qui guide les régulations on line de Grégoire au fil de la leçon. Ses interventions, adressées à un élève ou groupe d'élèves (triplette ou doublette selon les tâches), sont ciblées sur la parade (tâche 1), sur les techniques de réalisation du nœud d'assurage (tâche 2) ou sur l'assurage et le contreassurage (tâche 3). Lorsqu'il s'adresse au grimpeur, il désigne le plus souvent les conduites attendues du point de vue de la relation à l'assureur : Lorsque tu es en difficulté, tu dis sec, ça veut dire que t'es pas loin de lâcher! C'est plus rarement qu'il intervient sur les techniques de grimpe, dont l'objet concerne alors le choix des prises et surtout les appuis pieds : Pose tes pieds sur la paroi! Repousse la paroi avec tes pieds! Les jambes avant les bras!

L'extrait suivant, relatif aux données filmées d'un évènement remarquable tiré de l'acte 3 , met particulièrement en évidence les modalités de régulation et les préoccupations de Grégoire au cours de cette leçon :

Gr [à l'atelier traversées] : Les garçons, le départ c'est ici, jusqu'à la voie 8 ! Vous vous rappelez? Vous me faites deux traversées?

Kévin : Combien?

Paul : 8 !

Gr : Pour le moment, il n'y a pas de consigne, je donne une consigne après.

Gr [se dirige vers l'atelier nœuds] : Attention, Laura, ..., vas-y Laura, vers le bas [fait un geste de haut en bas pour montrer le trajet de la corde]

Gr [légèrement à distance d'Emilien qu'il regarde assurer] : Emilien, tu t'en sors ? 4 temps, en même temps! Emilien? Bras gauche, bras doit [mime le geste pour accompagner ses consignes], lorsque tu ramènes le bras gauche vers toi, le bras droit s'éloigne. Ensuite, non, on abaisse, sécurité, tu abaisses le bras droit !

Gr [se rapproche d'Emilien] : Non, ça, ça [accompagne Emilien dans la réalisation de son gestes d'assurage]. Et là, tu continues! Non, en bas toujours [mime en même temps]. Là, en bas! Regarde!

Cet extrait montre que Grégoire intervient sur le rappel de la tâche à l'atelier "ascension » alors qu'il s'attarde à redéfinir et accompagner (verbalement et par la démonstration) les techniques d'assurage de deux élèves à l'atelier «réalisation des nœuds». Dans l'entretien post-leçon, il explique le partage de ses interventions en relation avec sa focalisation sur les aspects sécuritaires, condition nécessaire, selon lui, pour permettre aux élèves de grimper :

$\mathrm{Au}$ final, [...] ce qui me préoccupait, c'est vraiment le fait de monter assuré, de valider le test de sécurité [...] Au moins je savais qu'en étant ici [à l'atelier réalisation de nœuds], si y' avait quelque chose à réguler chez un élève, je pouvais 
le faire dans l'instant, sachant qu'après je passais de groupe en groupe et qu'ils avaient le droit de grimper.

Le verbatim de l'entretien traduit aussi les tensions qui résultent de sa volonté d'être présent aux deux ateliers :

C'est vrai que j'étais, je me souviens maintenant, plus dans le coin ici et en paroles fort pour les autres en traversées... Donc j'ai les yeux ici et la voix là-bas, j’ai l'impression ! C'est la difficulté que j'ai éprouvée, je trouve. J'ai envie d'être partout pour voir et j'essaie aussi d'être en aide pour ceux qui sont le plus en difficulté, notamment au niveau sécurité.

Finalement, lors de cette leçon, les actions de l'ES consistent à définir et organiser le déroulement des situations, à désigner ponctuellement les comportements sécuritaires attendus et à les valider à l'aide d'une fiche. Ses gestes sont donc pilotés par une double préoccupation : organiser l'avancée du temps didactique en définissant les tâches et en contrôlant leur réalisation (chronogenèse); maintenir un espace sécuritaire et collaboratif de travail lié aux rôles d'aide au grimpeur (assurage, contre-assurage, parade).

\subsection{Les gestes de Grégoire lors de la leçon de volley-ball}

\subsubsection{Les gestes rattachés aux tâches d'organisation et de conception des dispositifs d'étude}

Le synopsis de la leçon de volley-ball (tableau 2), la troisième du cycle, montre qu'elle s'organise autour quatre types de tâches: a) un échauffement spécifique qui se décompose en trois tâches dont l'objectif est l'appropriation de techniques gestuelles; $b$ ) une situation problème $e^{5}$ c) une tâche mettant en jeu les savoirs mis en problèmes dans la tâche précédente; et d) une situation de jeu 4 contre 4 , qui correspond à la situation de référence du cycle (entretien post-leçon). 
Tableau 2 : Synopsis de la leçon 3 de volley-ball.

\begin{tabular}{|c|c|c|}
\hline 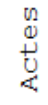 & $\begin{array}{l}\text { Activité du professeur } \\
\text { (Gr) et des élèves }\end{array}$ & Types de tâches \\
\hline$\approx$ & $\begin{array}{l}\text { Gr définit les objectifs de } \\
\text { séance puis désigne deux élèves } \\
\text { chargés de mener. Il fait un } \\
\text { schéma de la situation } 2 \text { au } \\
\text { tableau pendant que deux élèves } \\
\text { qu'il a désignés mènent } \\
\text { l'échauffement dynamique pour }^{\prime} \text { toute la classe }\end{array}$ & Echauffement dynamique \\
\hline $\mathcal{X}$ & $\begin{array}{l}\text { Gr rassemble les élèves au } \\
\text { tableau puis définit la } \\
\text { situation 1a. Il définit avec } \\
\text { les élèves les critères de } \\
\text { réalisation de la passe haute } \\
\text { (situation la). pendant que les } \\
\text { élèves réalisent la tâche, il } \\
\text { installe des plots sur les } \\
\text { terrains tout en effectuant des } \\
\text { retours verbaux individuels }\end{array}$ & $\begin{array}{l}\text { Situation 1a: passe } \\
\text { haute à } 10 \text { doigts sans filet } \\
\text { Maintien de 1' échange en } \\
\text { passe haute à } 10 \text { doigts }\end{array}$ \\
\hline 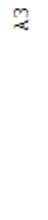 & $\begin{array}{l}\text { Gr rassemble les élèves au } \\
\text { milieu du gymnase, définit en } \\
\text { les questionnant les critères } \\
\text { de réalisation de la manchette } \\
\text { (situation 1b) puis démontre le } \\
\text { geste avec un élève en le } \\
\text { conmentant. Puis Gr annonce le } \\
\text { changement de rôles. }\end{array}$ & $\begin{array}{l}\text { Situation 1b: Manchette } \\
\text { sans filet } \\
1 \text { élève renvoie en } \\
\text { manchette et 1'autre en } \\
\text { passe haute (puis changement } \\
\text { de rôles) }\end{array}$ \\
\hline$\underset{z}{*}$ & $\begin{array}{l}\text { Gr rassemble les élèves au } \\
\text { milieu du gymnase, définit la } \\
\text { situation 1c puis démontre avec } \\
\text { un élève. Puis Gr fait des } \\
\text { retours individualisés. }\end{array}$ & $\begin{array}{l}\text { Situation 1c: } 1 \text { contre } 1 \\
\text { au-dessus du filet: } \\
\text { Départ ligne rouge, } 2 \\
\text { jonglages en avançant, } \\
\text { renvoi au partenaire et } \\
\text { replacement }\end{array}$ \\
\hline
\end{tabular}

¿ Gr rassemble les élèves au milieu du gymnase, définit la situation $1 \mathrm{c}$ puis démontre avec un élève. Puis Gr fait des retours individualisés.

2 a) Gr regroupe les élèves devant le tableau et définit la situation 2 puis $\mathrm{Gr}$ définit et répartit les groupes de travail sur les demi-terrains. Gr intervient individuellement auprès des élèves en passant de groupe en groupe

b) Gr regroupe les élèves en fin de tâche et les questionne sur la situation

2 a) Définit situation 3 en questionnant les élèves puis fait démontrer la tâche par un groupe et commente leurs réalisations ( 3 fois). Puis il intervient sur 2 groupes

b) Gr regroupe les élève, définit les problèmes rencontrés et institutionnalise les comportements attendus (mise en perspective leçon 4)
Situation 1c: 1 contre 1 au-dessus du filet :

Départ ligne rouge, 2 jonglages en avançant, renvoi au partenaire et replacement

Situation 2: attaque mashée 2 contre 1

a) Par 3, réaliser une attaque smashée, avec un défenseur qui engage, un réceptionneur qui fait une passe à l'attaquant qui smashe

b) Définition des comportements attendus : réalisation d'une "bonne passe » et synchronisation passeur attaquant (timing)

Situation 3: construction de $1^{\prime}$ attaque smashée

a) L'attaquant engage la balle en passe haute pour le passeur qui lui renvoie la balle pour qu'il smashe

b) définition des problèmes rencontrés : réalisation de la passe, identification $d^{\prime}$ une balle d'attaque, timing 


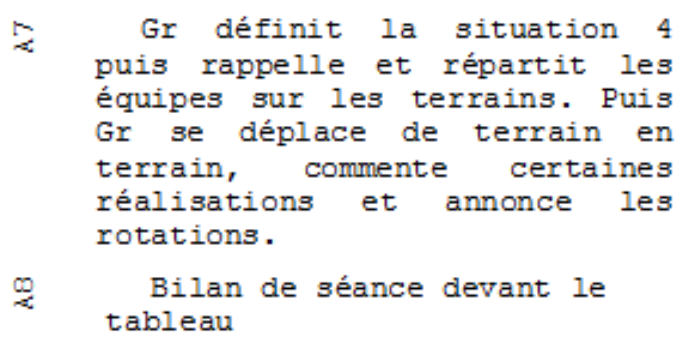
questionne les élèves et s'appuie sur leurs réponses (parfois à l'aide d'une démonstration qui les implique) pour : a) définir les critères de réalisation lors de la présentation des tâches; b) légitimer les comportements attendus, les savoirs en jeu lors des moments d'institutionnalisation (à la fin des tâches 2 et 3 , lors du bilan de séance). Les modalités de partage des responsabilités entre professeur et élèves sont liées à sa volonté d'impliquer les élèves dans leurs apprentissages tout en s'assurant de leur compréhension à travers des moments d'institutionnalisation afin de contrôler l'avancée du temps didactique.

\subsubsection{L'aide à l'étude pilotée par la construction progressive et problématisée de l'attaque smashée}

Lorsqu'il régule individuellement l'activité des élèves, Grégoire intervient sur différents registres, tels que les repères spatio-temporels, les techniques gestuelles, les trajectoires du ballon, en questionnant ou en commentant l'activité de l'élève : Essaie de te mettre ici, comme si le ballon était super chaud! Si la balle elle arrive là, pourquoi tu ne la prends pas en passe haute? Engage une balle plus haute pour lui laisser du temps! La démarche adoptée au cours de cette leçon repose sur l'utilisation d'expressions imagées (coiffer le ballon), de démonstrations (pour moi c'est peut-être un gain de temps plutôt que d'expliquer) et de séquences de verbalisation afin que les élèves analysent un peu et identifient leurs problèmes (entretien post-leçon). Le positionnement spatial de l'ES est particulièrement varié 
(proche du tableau, au milieu du gymnase, circulation dans un sens préétabli) et adapté à ses actions (définir, réguler ou institutionnaliser).

L'évènement remarquable (tiré de l'acte 6), dont sont extraites les données filmées restituées ci-dessous, traduit particulièrement les façons dont Grégoire conduit les échanges verbaux avec les élèves lors des moments d'institutionnalisation :

Gr : Selon vous, quels sont les problèmes rencontrés?

Arthur : Au niveau du passeur!

Gr: Au niveau du passeur?

Julien : Au niveau du moment où on part et de l'élan qu'on prend!

Rémi : La prise d'élan!

Gr: La prise d'élan?

Paul : On n'est pas synchro avec la passe !

Cassandra : Le timing!

Gr : Cassandra, tu as parlé de timing. Le problème du timing !

Julien : On n'est pas coordonné !

Gr : Voilà ! Le problème, c'est la synchronisation entre le passeur et l'attaquant. Donc, on va essayer de répondre à ce problème-là.

Gr [se rapproche du tableau] : La situation, elle va être simple. L'objectif : apprentissage du smash.

Puis Gr définit la tâche 3 [en utilisant le schéma du tableau (réalisé au cours de l'acte 1)].

Dans cet extrait, Grégoire prend appui sur les réponses des élèves pour faire émerger les contenus d'enseignement, relatifs aux repères spatio-temporels impliqués dans la réalisation du smash. Dans l'entretien post-leçon, il explicite sa démarche, préconisée en formation initiale, consistant à contextualiser, décontextualiser les apprentissages, puis questionne son efficacité :

Alors dans un premier temps, y'avait pas de consignes, c'était "construire une attaque smashée". Et là, on isole les problèmes qu'ils ont rencontrés, donc notamment dans un premier temps-là, un problème d'impulsion, d'endroit de passe et moment de déclenchement de la course d'élan pour aller smasher. Mais malheureusement, on s'aperçoit qu'en match on retrouve très peu ce qu'on a décontextualisé, on le retrouve très peu dans le contexte de jeu.

L'entretien révèle la place qu'il accorde aux moments de verbalisation et les tensions que ce choix induit (temps de pratique, compréhension versus réalisation) :

C'est long parce que, mine de rien, ils sont quand même 9 minutes sans pratiquer, là. Mais bon avec ce qu'ils avaient fait avant, ça me paraissait nécessaire de faire le bilan avec eux dans un premier temps des problèmes qu'ils avaient rencontrés. La verbalisation, elle reste importante parce que ça me permet de savoir s'ils ont au moins intégré les critères que je demande. Donc, ça veut dire qu'ils ont compris le geste, maintenant reste la réalisation!

récurrente, il explicite ses interventions et les dilemmes qu'il lui faut parfois résoudre en s'appuyant sur l'analyse des conduites des élèves référée aux enjeux d'apprentissage :

Et peut-être que elle (Julie) n'ira pas sur le smash en impulsion mais peut-être à une frappe de balle les pieds au sol, stabilisée. [...] Donc là c'est vrai qu'on va différencier le travail à ce niveau-là pour qu'elle puisse acquérir aussi, même si c'est pas la compétence d'une attaque smashée trajectoire descendante, au moins une attaque frappée avec une trajectoire tendue et peut-être placée si on y arrive. 
51 De manière générale, l'analyse qu'il fait de son intervention traduit les compromis qu'il effectue entre la poursuite de son objectif de leçon - construire une attaque smashée, une attaque accélérée comme disent les textes - et les modalités de différenciation mises en place pour que tous les élèves puissent réussir :

52 Alors, c'est ce que j'essaie de construire, c'est que entre guillemets plus forts jouent leur rôle au niveau des plus faibles, donc encouragement, coaching, etc. Dans la constitution des équipes, je cherche un rapport de force équilibré [...] et des équipes stables pour qu'ils aient un projet collectif ensemble (tâche 2).

53 En résumé, dans cette leçon, les gestes de Grégoire reposent sur l'alternance de régulations individualisées et collectives et sur l'orchestration de temps de verbalisation articulés à différents types de tâches (fermées, problème, de référence). Ses gestes relèvent de préoccupations multiples et résultent de compromis entre les modalités de conduite de l'étude (sollicitation de la verbalisation, de la compréhension des élèves) et l'avancée (collective mais aussi individuelle) du temps didactique.

\subsection{Analyse comparative des deux leçons}

54 Selon la perspective comparatiste adoptée (Mercier, Schubauer-Leoni \& Sensevy, 2002), nous souhaitons ici rendre compte de la singularité des manières dont l'ES met en actes le curriculum dans chacune des deux leçons observées mais également identifier des régularités qui, resituées dans leur contexte, permettraient d'éclairer des traits génériques de ses "gestes didactiques de métier ». L'analyse comparative est envisagée sous l'angle des processus de co-construction des savoirs puis des éléments qui les déterminent en arrière-plan.

\subsubsection{Processus de co-construction des savoirs}

Les deux leçons suivent une temporalité similaire, à savoir un échauffement collectif, des tâches ciblant un thème d'apprentissage, une tâche proche de la situation de référence puis un bilan collectif. Cette organisation respecte les intentions préalables de Grégoire définies au cours de l'entretien ante-cycle :

En fait, j'essaie d'avoir une situation de référence, que j'adapte et je mets des variables différentes de simplification et de complexification en fonction de mes différents niveaux de classe et des caractéristiques entre chaque classe.

Les façons dont sont partagées les responsabilités des transactions didactiques se spécifient au sein de chaque leçon en fonction des enjeux de savoir: en escalade, les processus de dévolution dépendent des contraintes sécuritaires; en volley-ball, ils sont totalement imbriqués à la mise en place des dispositifs d'apprentissage. Malgré ces divergences, que l'on peut expliquer par la spécificité de l'activité enseignée, les techniques topogénétiques de Grégoire sont conformes à son projet de classe, qui vise l' autonomie des élèves et leur responsabilisation au regard des différentes pratiques (entretien ante) et aux préconisations diffusées en formation initiale. Au-delà des spécificités organisationnelles liées au contexte, les similitudes constatées semblent attester du style (Clot, 1999) de l'ES, qui se renforce au fil des expériences d'enseignement ${ }^{6}$.

58 Aussi les manières dont l'ES met en actes le curriculum lors des deux leçons se différencient-elles surtout du point de vue de l'activité de guidage des apprentissages et de l'agencement des objets de savoirs au cours du temps. En effet, en volley-ball, la 
chronogenèse repose sur un enchaînement de dispositifs mettant progressivement à l'étude différents objets de savoir liés à la construction d'une attaque smashée, tandis que la continuité des objets de savoir dans les situations proposées en escalade s'avère moins visible en raison d'une focalisation importante sur les aspects sécuritaires (parade, assurage et contre-assurage). Et alors que les gestes d'aide à l'étude se caractérisent par des retours verbaux individualisés et ponctuels en escalade, ils relèvent en volley-ball de l'alternance d'interventions collectives et individualisées, centrées sur l'appropriation des enjeux de savoir par les élèves et associées à des modalités d'intervention variées (démonstration, positionnement spatial, modularité de l'intonation).

En conséquence, les résultats révèlent une évolution des processus de co-construction des savoirs entre les deux leçons. Si les gestes didactiques de métier de Grégoire sont dirigés par le pilotage des tâches et le maintien d'un espace collaboratif de travail (associé aux rôles de l'assurage) dans la leçon d'escalade, ils s'organisent autour de la progressivité et de la problématisation des apprentissages au cours de la leçon de volley-ball.

\subsubsection{L'influence de l'activité d'apprentissage des élèves, des références externes et institutionnelles}

C'est en croisant une analyse des actions de l'ES et des verbalisations d'entretiens que nous avons mis en évidence ses modalités d'analyse et de réflexivité dans et sur l'action, et partant son épistémologie pratique, qui rend compte des manières dont sont incorporés les savoirs dans ses « gestes didactiques de métier ».

61 Les résultats de cette étude confirment le rôle organisateur des prescriptions officielles chez les novices (Clot, 2008) et montrent les modalités dont l'ES les exploite pour choisir les savoirs mis à l'étude dans la classe :

Les programmes, pour moi, c'est plutôt une aide parce que lorsque je travaille par rapport à un objectif de classe, je prends toujours la compétence attendue, je prends les contenus d'enseignement, je retire les principaux contenus d'enseignement des fiches d'aide, les fiches ressources (Entretien post-recueil).

Ainsi, en escalade, les choix des savoirs enseignés sont guidés par les orientations définies dans les programmes officiels d'EPS, ce qui amène Grégoire à se focaliser sur les aspects sécuritaires : Par rapport aux compétences attendues, l'objet ça va être d'aller vers le bac. Donc ça va être la sécurité dans un premier temps du cycle puis grimper en moulinette, etc, pour aller au fur et à mesure vers grimper en tête (entretien ante).

Mais les façons dont l'ES conduit l'étude des savoirs dans sa classe révèlent également des processus de spécification des programmes liés aux difficultés rencontrées par certains élèves. Dans la leçon de volley-ball, l'ES se distancie parfois des prescriptions formalisées dans les programmes pour que tous les élèves puissent réussir, comme lorsqu'il accepte (pour Julie) la réalisation d'une attaque placée au lieu d'une attaque smashée telle que prescrite par les programmes (voir section 3.2.2). Plus généralement, l'analyse des propos de Grégoire au cours des entretiens post-leçons confirme l'évolution de son mode d'analyse et de réflexivité, qui se déplace d'une justification de ses choix au regard de la mise en œuvre des programmes à une explicitation de son activité orientée par l'interprétation des conduites effectives des élèves, de façon conforme aux modalités décrites par Grosstephan (2010). Ainsi, le regard de l'ES sur son métier se transforme : il se focalise davantage sur l'observation de l'activité d'apprentissage des élèves et anticipe leurs conduites dans les situations : 

qu'indicateurs des processus à l'œuvre dans l'élaboration du curriculum en actes en nous inscrivant dans une approche didactique à visée comparatiste, enrichie par les perspectives issues de la clinique de l'activité. C'est en raison des soubassements vygotskiens de l'interactionnisme social que peuvent être rapprochées les études de l'action conjointe en didactique (Amade-Escot \& Venturini, 2009; Venturini, 2012) et celles réalisées en clinique de l'activité (Amigues, 2003, 2009 ; Clot, 2008 ; Saujat, 2004).

71 Étudier le curriculum en actes suppose d'analyser les articulations entre curriculum prescrit, curriculum réalisé et pratiques externes. Dans cette perspective, le recours à la théorie de l'action conjointe en didactique permet de voir comment fonctionnent les références externes dans la classe puisque cette approche se donne pour projet d'accéder aux processus contextualisés de construction des savoirs en lien avec leurs fondements historico-culturels ${ }^{7}$. Par exemple, lorsque Grégoire, au cours de la leçon de volley-ball, intervient auprès d'une élève en difficulté (Cassandra) dans la réalisation de la passe, il 
questionne le " collé » en volley-ball en prenant appui sur la définition de cette pratique sociale de référence (voir section 3.2.3). Comme le soulignent de nombreux auteurs (Chevallard, 2010; Filliettaz \& Bronckart, 2004; Schubauer-Leoni et al., 2007), l'appropriation des savoirs scolaires ne peut être pensée indépendamment des œuvres et des pré-construits historico-culturels desquels ils proviennent. D'une certaine façon le curriculum en actes actualise le «référent construit » qui émerge au cours de l'action conjointe (Schubauer-Leoni et al., 2007, p. 56).

D'autre part, afin d'identifier le réel des gestes du professeur, et en particulier les décalages entre ses préoccupations, les injonctions institutionnelles et le curriculum réalisé, nous avons emprunté à la clinique de l'activité les méthodologies d'entretien en auto-confrontation (Clot, 1999). Pour autant, sans nier l'importance des usages des concepts sur l'augmentation du pouvoir d'action des enseignants débutants (Bertone, Chaliès \& Clot, 2009; Saujat, 2004), nous avons choisi de cibler notre étude sur les dimensions didactiques des gestes de l'ES, selon une visée compréhensive et non transformative. Conformément à cette orientation, les résultats de notre recherche confirment que l'ES s'approprie progressivement le métier en prenant appui sur les prescriptions officielles (descendantes au sens de l'ergonomie). Les processus spécifiques qu'il déploie pour mettre en œuvre les programmes sont marqués par des temporalités singulières, par l'agencement des objets de savoir dans les dispositifs, par la différenciation des contenus d'enseignement selon les élèves, par l'adaptation des formes de groupement et par des stratégies de dévolution accordant une place centrale à la verbalisation. Les prescriptions officielles, redéfinies en contexte par l'enseignant, sont donc considérées comme une ressource orientée vers les moyens d'action du professeur et vers l'activité des élèves (Amigues, 2009; Félix \& Saujat, 2008; Saujat, 2007). Nos résultats montrent également que les références socio-culturelles jouent le rôle d'interface entre l'activité des élèves et la mise en œuvre des programmes. Cette réappropriation personnelle par l'enseignant des préconstruits institutionnels et culturels dans l'action conjointe caractérise son épistémologie pratique et, au-delà, l'évolution de son rapport au métier.

73 Autrement dit, la mise en actes du curriculum par un enseignant lors de son entrée dans le métier procède des registres du métier décrits par Clot $(2008,2013)$ : a) le registre «impersonnel» lié aux prescriptions; b) le registre "personnel» qui relève de l'épistémologie pratique ${ }^{8} \mathrm{du}$ professeur, de son histoire, de ses appartenances institutionnelles; et c) le registre «interpersonnel » qui émane des ajustements réalisés au cours des interactions avec les élèves9. Selon Clot (2008, p. 262) : "le métier "en général" ne se dissout dans aucune des réalisations génériques qu'il traverse. Au contraire, il s'y réfracte et peut s'en dégager ». Les processus de mise en actes du curriculum traduisent également comment l'enseignant débutant construit son rapport au métier. L'originalité de notre approche est de montrer en quoi les processus didactiques de co-construction des savoirs sont à l'œuvre dans le développement du style de l'enseignant à travers l'appropriation du genre. De ce point de vue, le concept d'épistémologie pratique $d u$ professeur souligne particulièrement l'importance du contexte (la dimension située) dans la dynamique des rapports aux savoirs façonnés au fil de l'expérience. En pointant la circularité entre l'expérience stricto sensu de l'enseignant et les référents théoriques que le professeur mobilise ou (re)construit dans l'action conjointe, ce concept rend compte de la singularité des "gestes didactiques de métier » d'un enseignant. 
74 Finalement, l'analyse des "gestes didactiques de métier » permet de documenter les questions de métier sans renoncer à en saisir les dimensions didactiques.

\section{Conclusion}

75 L'enjeu de cette recherche était de décrire et comprendre les " gestes didactiques de métier » d'un enseignant stagiaire en EPS lors de son insertion professionnelle afin de caractériser les processus en jeu dans la construction du curriculum en actes.

Les traces recueillies et la temporalité de cette étude nous ont permis d'identifier les traits saillants de ses « gestes didactiques de métier » à travers une étude comparative de deux leçons effectives d'enseignement menées dans deux activités à différents moments de l'année. Les résultats révèlent une relative continuité des modes d'intervention de l'ES que nous avons attribuée à son style (Clot, 1999) et à son affirmation au fil de l'expérience. Les divergences mises en évidence concernent les manières d'agencer le milieu didactique en lien avec le découpage des objets de savoir dans la leçon ainsi que les modalités d'analyse et de réflexivité (Grosstephan, 2010). Reposant sur des préoccupations différentes, elles dépendent d'une part de la spécificité des activités physiques et sportives enseignées et d'autre part de l'évolution professionnelle du stagiaire.

En croisant une approche comparatiste en didactique et une analyse clinique de l'activité, nous avons cherché à caractériser les processus de co-construction des savoirs dans la classe en étudiant le réel des gestes didactiques de métier d'un enseignant stagiaire. Ces choix ont permis de mettre au jour l'imbrication de dimensions impersonnelles, personnelles et interpersonnelles du métier impliquées dans la mise en actes du curriculum par un enseignant débutant. Dans la continuité de ce travail, l'analyse des usages des prescriptions officielles au sein d'équipes pédagogiques pourrait contribuer à éclairer les dimensions transpersonnelles du métier et les manières dont elles influencent le curriculum en actes.

\section{BIBLIOGRAPHIE}

Amade-Escot, C. (2013). L'épistémologie pratique des professeurs et les recherches sur l'intervention. Perspectives pour de futurs dialogues. In B. Carnel \& J. Moniotte, Intervention, Recherche et Formation : quels enjeux, quelles transformations? (pp. 37-58). Actes du 7ème Colloque international ARIS.

Amade-Escot, C., Amans-Passaga, C., \& Montaud, D. (2009). Les savoirs mobilisés dans l'action didactique par les intervenants en sport : un entrelacs de processus épistémiques et institutionnels, Sciences de la société, 77, 43-62.

Amade-Escot, C., \& Dugal, J.P. (2010). Registre d'utilisation des savoirs didactiques en formation professionnelle des enseignants : le cas d'une conseillère pédagogique. Savoirs, 22, 108-131. 
Amade-Escot, C., \& Venturini, P. (2009). Le milieu didactique : d'une étude empirique en contexte difficile à une réflexion sur le concept. Education \& Didactique, 3(1), 7- 43.

Amigues, R. (2003). Pour une approche ergonomique de l'activité enseignante. Skolê (Hors-série), 1, 5-16.

Amigues, R. (2009). Le travail enseignant : prescriptions et dimensions collectives de l'activité. Les Sciences de l'éducation pour l'Ere nouvelle, 42 (2), 11-24.

Audigier, F., \& Tutiaux-Guillon, N. (Dir.) (2008). Compétences et contenus. Les curriculums en questions. Bruxelles : De Boeck.

Bertone, S., Chaliès, S., \& Clot, Y. (2009). Evaluer les pratiques réflexives en formation, Le travail humain, $72, n^{\circ} 2,104-125$.

Brière-Guenoun, F., \& Amade-Escot, C. (2010). Analyse in situ des savoirs mobilisés par un professeur d'éducation physique et sportive dans l'interaction didactique. Revue Suisse des sciences de l'éducation, 32 (2), 595-614.

Brière-Guenoun, F., \& Musard, M. (2012). Analyse didactique des gestes professionnels d'étudiants stagiaires en éducation physique et sportive. Revue des sciences de l'éducation, 38 (2), 275-301.

Brière-Guenoun, F., \& Reffugi, R. (2007). Organiser un chemin pour l'apprentissage. In C. AmadeEscot (Éd.), Le Didactique (pp. 67-82). Paris : Revue EPS, collection « Pour l'action ».

Chevallard, Y. (1997). Familière et problématique, la figure du professeur. Recherche en Didactique des Mathématiques, 17/3, 17-54.

Chevallard, Y. (1999). L'analyse des pratiques enseignantes en théorie anthropologique du didactique. Recherche en Didactique des Mathématiques, 19/2, 221-266.

Chevallard, Y. (2003). Approche anthropologique du rapport au savoir et didactique des mathématiques. In S. Maury \& M. Caillot (Dir.), Rapport au savoir et didactique (pp 81-104). Paris, Editions Fabert,

Chevallard, Y. (2010). La didactique, dites-vous ? Education \& didactique, 4(1), 139-146.

Clot, Y. (1999). La fonction psychologique du travail. Paris : Presses universitaires de France.

Clot, Y. (2008). Travail et pouvoir d'agir. Paris : Presses universitaires de France.

Clot, Y. (2013). Développer le métier : le collectif dans l'individu. In B. Carnel \& J. Moniotte, Intervention, Recherche et Formation : quels enjeux, quelles transformations? (pp. 24-36). Actes du 7ème Colloque international ARIS.

Devos-Prieur, O. (2011). Chronogenèse des objets enseignés en basket-ball par quatre intervenants à l'école primaire. eJRIEPS, 24, 118,143.

Félix, C., \& Saujat, F. (2008). L'aide au travail personnel des élèves entre déficit de prescriptions et « savoirs méthodologiques » : un double regard didactique et ergonomique. In Analyse des situations didactiques : perspectives comparatistes, Dossiers des Sciences de l'Education, 20, 125-138.

Filliettaz, L., \& Bronckart, J-P. (2004). La construction des significations dans l'activité située. L'exemple des rapports entre gestualité et langage en situation de travail. In C. Moro \& R. Rickenmann (Eds), Situation éducative et significations (pp. 35-58). Bruxelles : De Boeck, collection Raisons éducatives. 
Grosstepan, V. (2010). Développement professionnel d'enseignants d'EPS : processus et effets dans le cadre d'un dispositif de formation continue associant praticiens et chercheur. Thèse de doctorat inédite, Université de Reims.

Leriche, J. (2010). Analyse didactico-écologique des pratiques d'accompagnement de stagiaires en éducation physique au Québec et en France à partir de deux études de cas. Thèse de doctorat inédite, Université de Sherbrooke (Québec) et Université Paul Sabatier de Toulouse (France).

Leutenegger, F. (2009). Le temps d'instruire. Approche clinique et expérimentale du didactique ordinaire en mathématique. Berne : Peter Lang.

Mercier, A., Schubauer-Leoni, M-L., \& Sensevy, G. (2002). Vers une didactique comparée. Revue française de pédagogie, 141, 5-16.

Monnier, N., \& Amade-Escot, C. (2009). L'activité didactique empêchée : outil d'intelligibilité de la pratique enseignante en milieu difficile. Revue française de pédagogie, 168, 59-73.

Saujat, F. (2004). Comment les enseignants débutants entrent dans le métier. Formation et pratiques d'enseignement, 1, 97-106.

Saujat, F. (2007). Enseigner : un travail. In V. Dupriez \& G. Chapelle (Eds.). Enseigner (pp. 179-188). Paris : PUF.

Schubauer-Leoni, M.-L. (2008). La construction de la référence dans l'action conjointe professeurélève. In N. Wallian, M. P. Poggi \& M. Musard (Éds.), Co-construire des savoirs : les métiers de l'intervention par les APSA (pp. 67-86). Besançon : PUFC.

Schubauer-Leoni, M-L., \& Leutenegger F. (2002). Expliquer et comprendre dans une approche clinique/expérimentale du didactique ordinaire. In F. Leutenegger \& M. Saada-Robert (Eds), Expliquer et comprendre en sciences de l'éducation (pp. 227-251). Paris, Bruxelles : De Boeck.

Schubauer-Leoni, M.-L., \& Leutenegger, F. (2005). Une relecture des phénomènes transpositifs à la lumière de la didactique comparée. Revue Suisse des sciences de l'éducation, 27(3), 407-429.

Schubauer-Leoni, M-L., Leutenegger, F. \& Ligozat, F., \& Flückiger, A. (2007). Un modèle de l'action conjointe professeur-élèves : les phénomènes didactiques qu'il peut/doit traiter. In G. Sensevy \& A. Mercier (Eds.), Agir ensemble : l'action didactique conjointe du professeur et des élèves (pp. 51-91). Rennes, France : PUR.

Sensevy, G. (2007). Des catégories pour décrire et comprendre l'action didactique. In Sensevy, G. \& Mercier, A. (2007). Agir ensemble : l'action didactique conjointe du professeur et des élèves (pp. 13-49). Rennes : PUR.

Sensevy, G., \& Mercier, A. (2007). Agir ensemble : l'action didactique conjointe du professeur et des élèves. Rennes : PUR.

Toullec-Théry, M., \& Marlot, C. (2013). Les déterminations du phénomène de différenciation didactique passive dans les pratiques d'aide ordinaire à l'école élémentaire. Revue française de pédagogie, 182, 41-54.

Venturini, P. (2012). Action, activité, « agir » conjoints en didactique : discussion théorique, Education et didactique, 1(6), 127-136.

Vinson, M., \& Dugal, J-P. (2011). De la formation continue à la formation initiale : observation du didactique et effets de formation. STAPS, 91, 63-76. 


\section{NOTES}

1. La loi d'orientation et de programmation pour la refondation de l'École de la République, définitivement adoptée par le Parlement le 25/06/2013, se donne pour finalité de créer les conditions de l'élévation du niveau de tous les élèves et de la réduction des inégalités.

2. Nous avons jugé nécessaire de laisser le temps à l'ES de s'intégrer dans l'établissement et d'installer sa présence dans ses classes avant de mettre en place le dispositif de recherche.

3. La cinquième séance du cycle d'escalade (fin janvier) a aussi été enregistrée mais pour des raisons de place, nous ne développerons que deux leçons (la première et la dernière enregistrées) dans le cadre de cet article.

4. À la différence de Leutenegger et Schubauer-Leoni, les évènements remarquables ne reposent pas seulement sur les données d'enregistrement filmé.

5. Terme employé par Grégoire dans l'entretien post-leçon et au cours de la présentation de la tâche avec les élèves.

6. Les deux leçons sont espacées de quatre mois.

7. Dont certaines dimensions ne sont pas forcément pointées dans les programmes.

8. Parce qu'elle rend compte des manières dont l'enseignant fait vivre en situation des savoirs tirés des pratiques socio-culturelles (Amade-Escot, 2013b ; Schubauer-Leoni et al., 2007).

9. Le registre «transpersonnel » relevant de l'histoire collective est indirectement lié aux autres registres mais ne constitue pas une dimension prioritairement explorée dans cette étude (en raison des méthodologies développées).

\section{RÉSUMÉS}

Cette recherche vise à rendre compte de la dynamique du curriculum en actes à partir d'une analyse des " gestes didactiques de métier » d'un enseignant stagiaire d'EPS. Elle s'intéresse aux manières dont ce dernier met à l'étude les savoirs dans l'interaction didactique dans deux leçons à différents moments de l'année. La méthodologie croise des données d'enregistrements filmés et d'entretiens. L'analyse comparative des gestes de l'enseignant stagiaire lors des deux leçons révèle des divergences attribuées à la spécificité des activités enseignées ainsi qu'à l'affirmation de son style au fil de l'expérience. Au-delà, les résultats témoignent d'une réappropriation personnelle par l'enseignant des préconstruits institutionnels et culturels dans l'action conjointe. La discussion envisage les choix méthodologiques et théoriques opérés pour étudier la mise en actes du curriculum.

This research aims to account for the dynamics of the curriculum in acts through an analysis of "gestes didactiques de métier" of a physical education student teacher. It concerns the ways in which the teacher leads the study of knowledge in the didactic interaction in two lessons at different times of the year. The method connects video and interview data. Comparative analysis of the student teacher's gesture during two lessons shows differences which are attributed to the specificity of physical activities and the affirmation of the teacher's style over experience. Beyond, the results show a personal reappropriation by the teacher of institutional and cultural 
preconstructed in the joint action. Theoretical and methodological choices made in order to study the implementation of curriculum are finally discussed.

INDEX

Keywords : didactic, joint action, professional acts, physical education, student teacher Mots-clés : action conjointe, enseignant stagiaire, éducation physique et sportive, « gestes didactiques de métier »

\section{AUTEUR}

\section{FABIENNE BRIÈRE-GUENOUN}

Maître de conférences en sciences de l'éducation, Laboratoire Interdisciplinaire de Recherche sur les Transformations des pratiques Éducatives et des pratiques Sociales, LIRTES (EA 7313),

Université Paris-Est Créteil 